\title{
Load Frequency Control of Multi-Region Interconnected Power Systems with Wind Power and Electric Vehicles Based on Sliding Mode Control
}

\author{
Zhenghao Wang ${ }^{1}$, , Yonghui Liu ${ }^{1, *}$, Zihao Yang $^{2}$ and Wanhao Yang ${ }^{1}$ \\ 1 College of Electrical Engineering, Shanghai Dianji University, Shanghai 201306, China; \\ 196001010115@st.sdju.edu.cn (Z.W.); 186001010415@st.sdju.edu.cn (W.Y.) \\ 2 College of Electrical and Electronic Engineering, North China Electric Power University, \\ Beijing 102206, China; zihaoo@163.com \\ * Correspondence: liuyh@sdju.edu.cn; Tel.: +86-180-0186-2463
}

Citation: Wang, Z.; Liu, Y.; Yang, Z.; Yang, W. Load Frequency Control of Multi-Region Interconnected Power Systems with Wind Power and Electric Vehicles Based on Sliding Mode Control. Energies 2021, 14, 2288 https://doi.org/10.3390/en14082288

Academic Editors: Seon-Ju Ahn and Hyun-Koo Kang

Received: 6 March 2021

Accepted: 12 April 2021

Published: 19 April 2021

Publisher's Note: MDPI stays neutral with regard to jurisdictional claims in published maps and institutional affiliations.

Copyright: (c) 2021 by the authors. Licensee MDPI, Basel, Switzerland. This article is an open access article distributed under the terms and conditions of the Creative Commons Attribution (CC BY) license (https:// creativecommons.org/licenses/by/ $4.0 /)$.

\begin{abstract}
In recent years, wind power systems have been used extensively, which not only improve the efficiency of current conventional power generation systems, but also can save traditional fossil fuel resources. However, considering the instability of wind power, after being grid connected, it can easily cause an impact on the stability of the grid operation. Considering the above problems, this paper considers to make full use of the energy storage part of electric vehicles (EVs) to increase the stability of grid operation. Based on the mathematical model, this paper studies the load frequency control (LFC) problem of a multi-region interconnected power system with wind power and EVs. First, since the system states are difficult to be monitored, a state observer is designed to estimate the state. Based on this, the integral sliding mode controller (SMC) is designed to realize the LFC of the interconnected power system. Meanwhile, to obtain better control performance, this paper further analyzes and optimizes the controller parameters based on Lyapunov stability theory. At last, simulations are carried out for the power systems with two regions in Simulink. The results show that the designed controllers are effective to compensate the load demand disturbances. In addition, it is demonstrated that the battery storage of EVs can play the role of peak-shaving and valley-filling in LFC.
\end{abstract}

Keywords: multi-region interconnected power system; frequency control; state observer; integrated sliding mode control

\section{Introduction}

With the development of grid technology, communication technology is gradually integrated into the grid. As the connection between different regions is strengthened, a multi-regional interconnected power system is gradually formed in [1,2]. Multi-region interconnection can realize the economic operation of power system by coordinating different generation costs among different regions. Meanwhile, the interconnected areas can support each other with the help of tie-line, which improves the reliability of power supply. However, the multi-regional interconnection of power systems also has some unavoidable problems. Failures in the operation of power generation equipment and instability of renewable energy sources tend to cause fluctuations in the generation process. The fluctuations may affect the stability of the entire power system frequency through the tie-line [3,4]. Therefore, under the complex environment, more stringent standards are proposed for the stable operation of modern power systems. For power generation, integration of various energy sources is to be accomplished. Meanwhile, it is essential to ensure the safety and the efficiency of the transmission in electricity. More importantly, to ensure the control performance, it demands higher requirements for LFC in interconnected power systems. 
With the increasing maturity of the wind power technology, wind power has been widely used in many forms of renewable energy power generation. However, the randomness and uncertainty of wind energy tends to cause wind abandonment and load shedding. This phenomenon causes the imbalance of supply and demand of the system power, which may bring in the deviation of the power system frequency. With the large-scale wind power integration into the grid, it will affect the stability of the grid frequency from a certain extent [5-7]. Therefore, some scholars have researched the LFC of the power system after the grid connection of wind power in recent years. In order to track the stochastic fluctuations of wind power output, a model for decentralized load frequency prediction control of interconnected grids containing the wind farms was proposed in [8]. In [9], a decentralized SMC is designed to suppress the fluctuation of load frequency based on the construction of a power system model containing wind power. A robust LFC strategy based on event-driven communication is proposed in [10]. While reducing the transmission volume of network communication, the frequency stability of the power system is ensured. In [11], an online reinforcement learning method based on an adaptive dynamic model is proposed. It is shown that the method can suppresses the uncertainty caused by the large-scale access of wind power effectively.

Considering the uncertainty of wind power forecasting, a distributed demand-side management approach for smart grids is proposed in [12]. Through game-theoretic approach, the cost of consumer participation in demand-side management programs is minimized. According to the research mentioned in the above literature, it is mainly to adopt appropriate control strategies to solve the effect of wind power uncertainty on the system load frequency. However, considering the limitations of only relying on the controller to ensure system stability, we further consider supplementing the energy storage component to improve the robustness of the control system.

In addition, with the popularization of the concept of environmental protection, the market share of the electric vehicles (EVs) is rising year by year. According to statistics, except for the demand of daily traffic, nearly $90 \%$ of the EVs are not used. Therefore, LFC of power system with EVs has become a key research topic for researchers in recent years. The scale effect of EVs concentration is analyzed in [13]. It is verified that EVs energy storage can provide backup energy storage for the systems. In [14], a LFC model for the implementation of the controlled energy dynamic changes in EVs is constructed. The simulation shows that the EVs are able to switch between power and controllable load. Although the literature $[13,14]$ has investigated the mode of electric vehicles, the model of wind power and electric vehicles both connected to the grid is still open. To solve the problems of inaccurate mode of EVs entry and high anti-interference requirements, a linear self-interference control method is proposed in [15]. Considering the influence of the droop control characteristics of EVs, a combined optimization method of EVs-assisted frequency control and secondary frequency control of conventional units is proposed in [16]. The literatures $[15,16]$ focus on control performance of electric vehicles after grid integration. However, in the system that contains wind power and EVs, more effective control strategies need to be considered to improve the system performance.

Through the above analysis, it is found that when considering the system containing both wind power and electric vehicle, the problems of system model building and control strategy selection still need to be paid more attention. Meanwhile, the point should also be noted that, to reduce fluctuations in the frequency of the load, the traditional energy storage methods such as batteries are used in general. It not only increases the economic cost of grid construction, but also fails to make full use of the energy storage capacity of idle EVs. In addition, when the system suffers from load demand disturbances, the load frequency is also affected and fluctuates. For complex systems with perturbations, sliding mode control can effectively overcome effects of perturbations.

Therefore, to reduce the deviation of load frequency after area interconnection, it is necessary to study the LFC of the complex system containing wind power and EVs using the sliding mode control. 
Motivated by the above discussion, the contribution of this work is specified as follows:

i. After the wind power is connected to the grid, to solve the impact of its uncertainty on the system frequency stability, the energy storage of idle EVs is considered as the buffer in this paper. To study the LFC of grid, when the system suffers from load demand perturbation, the model of the system containing wind power and EVs is developed.

ii. In practice, the state is difficult to be grasped due to the limitation of equipment. Based on the model built in (I), the state observer is designed in this paper.

iii. SMC is an effective control method to deal with system disturbance. Therefore, in this paper, to achieve the goal of load frequency control, the integral sliding mode controller is designed.

iv. By combining the Lyapunov stability theory, the parameters of the controller are further optimized. In this way, better control performance of both the error system and the observer is ensured in the paper.

In this paper, the structure of the paper can be organized into five sections. First, we describe the background and motivation of this paper in Introduction of the Section 1. Then, the Section 2 presents description of the model building. In the Section 3, we focus on the theoretical analysis. The Section 4 includes the simulation results and the analysis. Finally, through simulation verification, we summarize the conclusions in Section 5.

\section{System Model and Description}

\subsection{Wind Power and EVs Model}

For wind power systems, the main issue is the active power output. Therefore, the wind power dynamics model is established as follows [17]:

$$
\Delta \dot{P}_{\mathrm{WTG} i}=\frac{1}{T_{\mathrm{WTG} i}} \Delta P_{\mathrm{w}-i}-\frac{1}{T_{\mathrm{WTG} i}} \Delta P_{\mathrm{WTG} i}
$$

where $\Delta P_{\mathrm{WTG} i}$ is the wind turbine output power deviation, $T_{\mathrm{WTG} i}$ is the wind turbine time constant, and $\Delta P_{\mathrm{w}-i}$ is the power of wind.

Normally, EVs tend to play the role of getting electricity from the grid. Benefit from the development of V2G technology, EVs energy storage enables participation in LFC. The main idea is to make full use of the battery energy when the EVs are non-working; to use it as a buffer for the fluctuations caused when renewable energy is integrated into the grid. But EVs cannot be integrated into the grid randomly. It not only needs to be managed centrally for EVs energy storage, but also combined with intelligent control between the grid. The control input of the EV part of this paper is expressed as:

$$
u_{\mathrm{e} i}(t)=\alpha_{\mathrm{e} i} u(t)
$$

where $\alpha_{\mathrm{e} i}, u(t)$ denotes EVs scale factor, control input, respectively.

To model EVs, the literature [18] focuses on the analysis of when EVs are connected to the grid. Meanwhile, to prevent the EVs charging and discharging simultaneously, the paper supplements the constraint. Instead, by establishing mathematical models, ref. [19] adequately equates the output power of EVs as energy storage part. To realize the control objective, considering the partial output of EVs, the EVs dynamics model is written in the following form [19]:

$$
\Delta \dot{P}_{\mathrm{e} i}=-\frac{1}{T_{\mathrm{e} i}} \Delta P_{\mathrm{e} i}+\frac{\alpha_{\mathrm{e} i} k_{\mathrm{e} i}}{T_{\mathrm{e} i}} u(t)
$$

where $\Delta P_{\mathrm{e} i}$ is the deviation of EVs output power, $k_{\mathrm{e} i}$ is EVs gain factor, and $T_{\mathrm{e} i}$ is EVs time instants. 


\subsection{Load Frequency Control Model with Wind Power and EVs}

Interconnected power systems usually consist of multiple control areas. Each area is connected to another area by tie lines to overcome the mismatch between generation and demand. In traditional LFC systems, automatic generation control acts as the secondary controller to control the grid frequency. To solve the instability caused by the wind power in the grid in recent years, some new topology structure of the interconnected power system is established after the introduction of electric vehicles, which is shown in Figure 1.

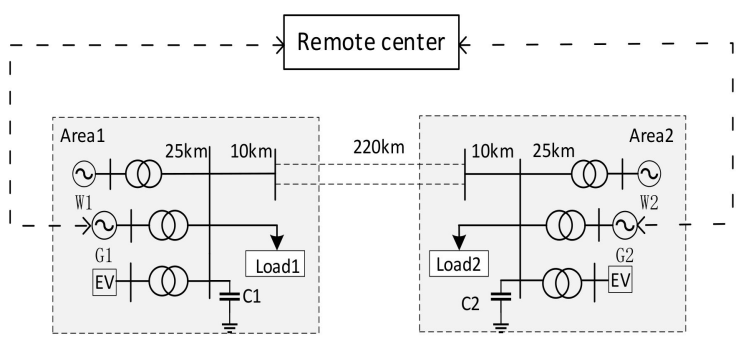

Figure 1. Topology of two-region power system including wind power and electric vehicles (EVs).

Under the stable operation of the power system, the system is linearized and simplified. In this model, a generator is used to represent the overall performance of the generator set. In addition, the time delay phenomenon of the control signal is ignored. Therefore, mathematical models containing the wind power and EVs power systems are shown in Figure 2 .

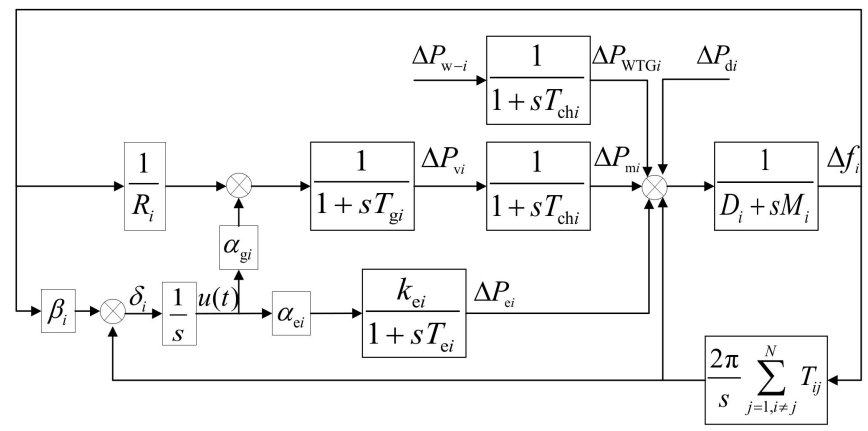

Figure 2. System load frequency control (LFC) model including wind power and EVs.

According to the system model established in Figure 2, the mathematical model of the system can be expressed as:

$$
\begin{aligned}
& \Delta \dot{f}_{i}=-\frac{D_{i}}{M_{i}} \Delta f_{i}+\frac{1}{M_{i}} \Delta P_{\mathrm{m} i}-\frac{1}{M_{i}} \Delta P_{\mathrm{tie}-i}+\frac{1}{M_{i}} \Delta P_{\mathrm{WTG} i}+\frac{1}{M_{i}} \Delta P_{\mathrm{e} i}-\frac{1}{M_{i}} \Delta P_{\mathrm{d} i} \\
& \Delta \dot{P}_{\mathrm{m} i}=-\frac{1}{T_{\mathrm{ch} i}} \Delta P_{\mathrm{m} i}+\frac{1}{T_{\mathrm{ch} i}} \Delta P_{\mathrm{v} i} \\
& \Delta \dot{P}_{\mathrm{v} i}=-\frac{1}{R_{i} T_{\mathrm{g} i}} \Delta f_{i}-\frac{1}{T_{\mathrm{g} i}} \Delta P_{\mathrm{v} i}+\frac{\alpha_{\mathrm{g} i}}{T_{\mathrm{g} i}} u(t) \\
& \Delta \dot{P}_{\mathrm{tie}-i}=2 \pi \sum_{j=1, i \neq 1}^{N} T_{i j} \Delta f_{i} \\
& \delta_{i}=\beta_{i} \Delta f_{i}+\Delta P_{\mathrm{tie}-i} \\
& \Delta \dot{P}_{\mathrm{WTG} i}=\frac{1}{T_{\mathrm{WTG} i}} \Delta P_{\mathrm{w}-i}-\frac{1}{T_{\mathrm{WTG} i}} \Delta P_{\mathrm{WTG} i} \\
& \Delta \dot{P}_{\mathrm{e} i}=-\frac{1}{T_{\mathrm{e} i}} \Delta P_{\mathrm{e} i}+\frac{\alpha_{\mathrm{e} i} k_{\mathrm{ei}}}{T_{\mathrm{e} i}} u(t)
\end{aligned}
$$

where $\Delta f_{i}$ is the frequency deviation, $\Delta P_{\mathrm{m} i}$ is the generator power deviation, $\Delta P_{\mathrm{v} i}$ is the control valve position deviation, $\Delta P_{\text {tie }-i}$ is the tie-line power exchange, $\delta_{i}$ is the area control deviation, $M_{i}$ is the rotational inertia of the generator set, $D_{i}$ is the load damping coefficient, $T_{\mathrm{g} i}$ is the governor time constant, $T_{\mathrm{ch} i}$ is the turbine time constant, $R_{i}$ is the governor droop characteristic, $\beta_{i}$ is the frequency deviation factor, $\Delta P_{\mathrm{d} i}$ is the load demand disturbance, 
and $\alpha_{\mathrm{g} i}$ is the turbine proportionality factor. The summary table of nomenclature is shown in Appendix A.

By simplifying the above equation of state, the system can be further expressed as:

$$
\begin{aligned}
& \dot{x}(t)=A \boldsymbol{x}(t)+B u(t)+D \boldsymbol{f}(t), \\
& \boldsymbol{y}(t)=\boldsymbol{C} \boldsymbol{x}(t),
\end{aligned}
$$

with

$$
\begin{gathered}
\boldsymbol{x}(t)=\left[\boldsymbol{x}_{1}(t) \boldsymbol{x}_{2}(t) \boldsymbol{x}_{3}(t) \cdots \boldsymbol{x}_{n}(t)\right]^{\mathrm{T}}, \\
\boldsymbol{y}(t)=\left[\boldsymbol{y}_{1}(t) \boldsymbol{y}_{2}(t) \boldsymbol{y}_{3}(t) \cdots \boldsymbol{y}_{n}(t)\right]^{\mathrm{T}}, \\
\boldsymbol{x}_{i}(t)=\left[\Delta f_{i} \Delta P_{\mathrm{m} i} \Delta P_{\mathrm{v} i} \Delta P_{\mathrm{tie}-i} \int \delta_{i} \Delta P_{\mathrm{WTG} i} \Delta P_{\mathrm{e} i}\right]^{\mathrm{T}}, \\
\boldsymbol{y}_{i}(t)=\left[\Delta f_{i} \int \delta_{i}\right]^{\mathrm{T}}, \\
\boldsymbol{f}(t)=\left[\Delta P_{\mathrm{d} i} \Delta P_{\mathrm{w}-i}\right]^{\mathrm{T}},
\end{gathered}
$$

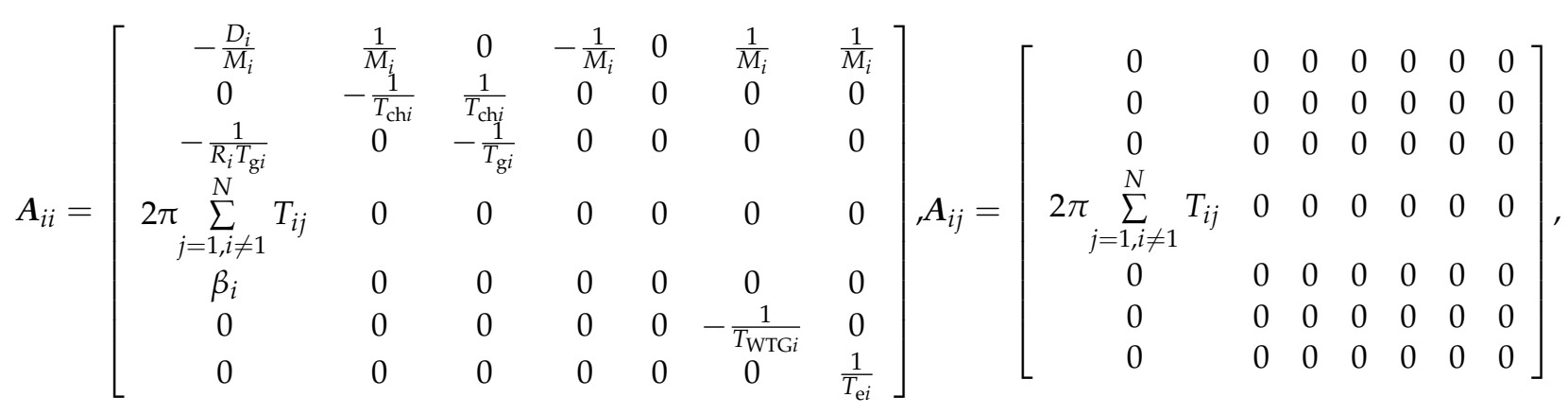$$
\boldsymbol{B}=\left[\begin{array}{c}
0 \\
0 \\
\frac{\alpha_{\mathrm{g} i}}{T_{\mathrm{g} i}} \\
0 \\
0 \\
0 \\
\frac{\alpha_{\mathrm{e} i} k_{\mathrm{e} i}}{T_{\mathrm{e} i}}
\end{array}\right], \boldsymbol{C}=\left[\begin{array}{ccccccc}
1 & 0 & 0 & 0 & 0 & 0 & 0 \\
0 & 0 & 0 & 0 & 1 & 0 & 0
\end{array}\right], \boldsymbol{D}=\left[\begin{array}{cc}
-\frac{1}{M_{i}} & 0 \\
0 & 0 \\
0 & 0 \\
0 & 0 \\
0 & 0 \\
0 & \frac{1}{T_{\mathrm{WTG} i}} \\
0 & 0
\end{array}\right],
$$

where $\boldsymbol{x}(t)$ is system state, $\boldsymbol{A}$ is state matrix, $\boldsymbol{B}$ is system input matrix, $\boldsymbol{C}$ is output matrix, $\boldsymbol{y}(t)$ is system output matrix, $u(t)$ is control signal, and $\boldsymbol{D}$ is coefficient matrix.

In this paper, we consider the effect of the disturbance component on the system. The disturbance part mainly has two parts: load demand disturbance and wind power magnitude. To help the derivation of the subsequent theory, assume that the perturbation considered in this paper is bounded, and it can be written as:

$$
\|f(t)\| \leq L_{i}
$$

where $L_{i}$ is a known constant.

\section{Model Characterization}

In this section, based on the model, we focus on the theoretical analysis of the state observer, the SMC, and the stability of the system. The flow chart is shown in Figure 3. 


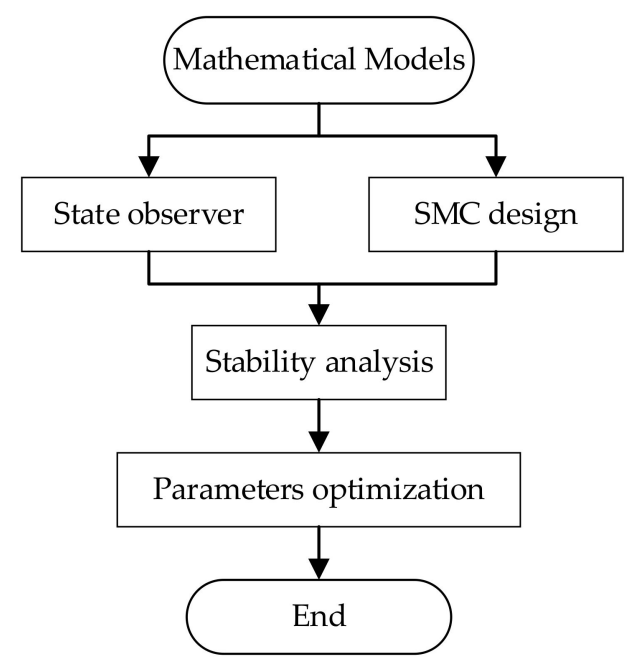

Figure 3. Flow chart of Section 3.

\subsection{Observer Design}

Since the integration of wind power and EVs, the complexity of the system model becomes complex. Meanwhile, due to the limitation of monitoring equipment and cost in engineering, the system state variable may not be accurately measured. To solve this problem, the state observer is designed as follows:

$$
\begin{aligned}
& \dot{\hat{x}}(t)=A x(t)+B u(t)+\boldsymbol{O}(\boldsymbol{y}(t)-\hat{y}(t)), \\
& \hat{y}(t)=\hat{C} \hat{x}(t),
\end{aligned}
$$

where $O$ is the observer gain matrix.

Considering state observer (6), the following observer-based integral sliding surface is designed:

$$
s(t)=\hat{S} \hat{x}(t)-S \int_{0}^{t}(\boldsymbol{A}-\boldsymbol{B K}) \boldsymbol{x}(\tau) d \tau,
$$

where $S$ and $K$ are matrices of appropriate dimensions to be designed.

Define the error variable $\boldsymbol{e}(t)=\boldsymbol{x}(t)-\hat{x}(t)$. When the system state reaches the sliding surface, it can be obtained that:

$$
\begin{gathered}
\dot{s}(t)=0, \\
\dot{s}(t)=S B u(t)+S O C e(t)+S B K \hat{x}(t),
\end{gathered}
$$

Thus, the equivalent control can be written as:

$$
u_{\mathrm{eq}}(t)=-K x(t)-(S B)^{-1} \operatorname{SOCe}(t) .
$$

Substituting (10) into (6), the dynamic equation of the system is obtained as follows:

$$
\dot{\hat{x}}(t)=(A-B K) \hat{x}(t)+\hat{S O C e}(t),
$$

where $\hat{S}=\boldsymbol{I}-\boldsymbol{B}(\boldsymbol{S B})^{-1} \boldsymbol{S}$.

According to (5), (6), and (10), the error system is obtained as follows:

$$
\dot{\boldsymbol{e}}(t)=(\boldsymbol{A}-\boldsymbol{O C}) \boldsymbol{e}(t)+\boldsymbol{F} \boldsymbol{f}(t)+\boldsymbol{B}\left(u(t)-u_{\mathrm{eq}}(t)\right) .
$$

Based on the above analysis, the controller $u(t)$ is designed such that the LFC of the system (5) can be achieved. 


\subsection{Reachability Analysis}

To achieve the reachability of the sliding mode surface, a SMC is designed as follows:

$$
u(t)=-\hat{K} \hat{x}(t)-(S B)^{-1} \operatorname{SOC} e(t)-\mu(S B)^{-1} s(t)-v(S B)^{-1} \operatorname{sgn}(s(t)),
$$

where $\mu>0$ and $v>0$ are positive control parameters to be designed.

The Lyapunov function is chosen as:

$$
V(t)=\frac{1}{2} s^{\mathrm{T}}(t) s(t)
$$

The derivative of (14) can be written as:

$$
\dot{V}(t)=\frac{s^{\mathrm{T}}(t) \dot{s}(t)}{\|s(t)\|},
$$

Noting that $s(t) \operatorname{sgn}(s(t))=\|s(t)\|_{1} \geq\|s(t)\|$ and $s^{T}(t) s(t) \geq\|s(t)\|^{2}$. It can be obtained from (15) that

$$
\begin{aligned}
\dot{V}(t) & =\frac{s^{\mathrm{T}}(t)}{\|s(t)\|}(\dot{\hat{\boldsymbol{x}}}(t)-\boldsymbol{S}(\boldsymbol{A}-\boldsymbol{B} \boldsymbol{K}) \hat{\boldsymbol{x}}(t)) \\
& \leq-\mu V(t)-v .
\end{aligned}
$$

Considering (16), one further has

$$
V(t) \leq\left(V(0)+\frac{v}{\mu}\right) \mathrm{e}^{-\mu t}-\frac{v}{\mu} .
$$

Therefore, under controller (13), when $t \geq t^{*}=\mu^{-1} \ln \frac{\mu\|s(0)\|+v}{v}$ is satisfied, the observer system trajectory can reach the sliding surface in finite time and remain there.

\subsection{Stability Analysis}

The sliding mode dynamics of the systems (5) can be described as an augmented system as follows:

$$
\left(\begin{array}{l}
\dot{e}(t) \\
\dot{\hat{x}}(t)
\end{array}\right)=\left(\begin{array}{cc}
\boldsymbol{A}-\boldsymbol{O C} & 0 \\
\hat{S} \boldsymbol{O C} & \boldsymbol{A}-\boldsymbol{B} \boldsymbol{K}
\end{array}\right)\left(\begin{array}{c}
\boldsymbol{e}(t) \\
\hat{x}(t)
\end{array}\right)+\left(\begin{array}{c}
\boldsymbol{D} \\
0
\end{array}\right) f(t)+\left(\begin{array}{c}
0 \\
-\mu \nu(\boldsymbol{S B})^{-1}
\end{array}\right) s(t)+\left(\begin{array}{c}
0 \\
-v \boldsymbol{B}(\boldsymbol{S B})^{-1}
\end{array}\right) \operatorname{sgn}(s(t)) .
$$

Ignoring the variable structure part, system (18) can be expressed as:

$$
\left(\begin{array}{l}
\dot{e}(t) \\
\dot{\hat{x}}(t)
\end{array}\right)=\left(\begin{array}{cc}
A-O C & 0 \\
\hat{S} O C & A-B K
\end{array}\right)\left(\begin{array}{c}
e(t) \\
\hat{x}(t)
\end{array}\right)+\left(\begin{array}{c}
D \\
0
\end{array}\right) f(t)
$$

According to the Lyapunov theory, if there exist positive definite matrices $Q_{1}, Q_{2}, P_{1}$, and $\boldsymbol{P}_{2}$ that satisfy the following LMIs:

$$
\begin{aligned}
& \operatorname{sym}\left(\boldsymbol{P}_{1}(\boldsymbol{A}-\boldsymbol{O} \boldsymbol{C})\right)+\boldsymbol{Q}_{1}<0, \\
& \operatorname{sym}\left((\boldsymbol{A}-\boldsymbol{B} \boldsymbol{K}) \boldsymbol{P}_{2}\right)+\boldsymbol{Q}_{2}<0,
\end{aligned}
$$

then the stability of the system (19) can be guaranteed.

Considering the system (18), one has

$$
\begin{aligned}
& \boldsymbol{e}(t)=\mathrm{e}^{(\boldsymbol{A}-\boldsymbol{O C}) t} \boldsymbol{e}(0)+\int_{0}^{t} \mathrm{e}^{(\boldsymbol{A}-\boldsymbol{O C})(t-\tau)} \boldsymbol{D} \boldsymbol{f}(\tau) d \tau, \\
& \hat{\boldsymbol{x}}(t)=\mathrm{e}^{(\boldsymbol{A}-\boldsymbol{B} \boldsymbol{K}) t} \hat{\boldsymbol{x}}(0)+\int_{0}^{t} \mathrm{e}^{(\boldsymbol{A}-\boldsymbol{B} \boldsymbol{K})(t-\tau)}\left(-\mu \boldsymbol{B}(\boldsymbol{S B})^{-1} s(t)-v \boldsymbol{B}(\boldsymbol{S B})^{-1} \operatorname{sgn}(s(t))\right. \\
& +\hat{S O C e}(\tau)) d \tau \text {. }
\end{aligned}
$$


Since $\boldsymbol{A}-\boldsymbol{O C}$ and $\boldsymbol{A}-\boldsymbol{B K}$ are Hurwitz matrix, there exist positive values $\lambda_{\mathrm{e}}>0, \lambda_{\mathrm{o}}>$ $0, M_{1}$ and $M_{2}$ such that (22) holds. Moreover, the equation (21) can be rewritten as:

$$
\begin{aligned}
\|\boldsymbol{e}(t)\| & \leq M_{1} \mathrm{e}^{-\lambda_{\mathrm{e}} t}+M_{1} \int_{0}^{t} \mathrm{e}^{-\lambda_{\mathrm{e}}(t-\tau)}\|\boldsymbol{D}\|\|\boldsymbol{f}(\tau)\| d \tau \\
\|\hat{\boldsymbol{x}}(t)\| & \leq M_{2} \mathrm{e}^{-\lambda_{\mathrm{o}} t}+M_{2} \int_{0}^{t} \mathrm{e}^{-\lambda_{\mathrm{o}}(t-\tau)}\left(\left\|\mu \boldsymbol{B}(\boldsymbol{S} \boldsymbol{B})^{-1}\right\|\left(\left\|s(0)+\frac{v}{\mu}\right\| \mathrm{e}^{-\mu \tau}-\frac{v}{\mu}\right)\right. \\
& \left.+\left\|v \boldsymbol{B}(\boldsymbol{S} \boldsymbol{B})^{-1}\right\|+\|\hat{S} \boldsymbol{O C}\|\|\boldsymbol{e}(\tau)\|\right) d \tau .
\end{aligned}
$$

Considering $\|f(t)\| \leq L_{i}$. By combing (17) with (22), one further has

$$
\begin{aligned}
\|\boldsymbol{e}(t)\| & \leq M_{1} \mathrm{e}^{-\lambda_{\mathrm{e}} t}+M_{1} \int_{0}^{t} \mathrm{e}^{-\lambda_{\mathrm{e}}(t-\tau)}\|\boldsymbol{D}\|\|\boldsymbol{f}(\tau)\| d \tau \\
\|\hat{\boldsymbol{x}}(t)\| & \leq M_{2} \mathrm{e}^{-\lambda_{\mathrm{o}} t}+M_{2} \int_{0}^{t} \mathrm{e}^{-\lambda_{\mathrm{o}}(t-\tau)}\left(\left\|\mu \boldsymbol{B}(S \boldsymbol{B})^{-1}\right\|\left(\left\|s(0)+\frac{v}{\mu}\right\| \mathrm{e}^{-\mu \tau}-\frac{v}{\mu}\right)\right. \\
& \left.+\left\|v \boldsymbol{B}(\boldsymbol{S B})^{-1}\right\|+\|\hat{S} \boldsymbol{O C}\|\|\boldsymbol{e}(\tau)\|\right) d \tau .
\end{aligned}
$$

Taking the limit of (23), one has

$$
\begin{aligned}
& \lim _{t \rightarrow \infty}\|\boldsymbol{e}(t)\| \leq \frac{M_{1}\|\boldsymbol{D}\| L}{\lambda_{\mathrm{e}}}, \\
& \lim _{t \rightarrow \infty}\|\hat{x}(t)\|=\frac{M_{1} M_{2} \| \hat{\text { SOOC }\|\| \boldsymbol{D} \| L}}{\lambda_{\mathrm{o}} \lambda_{\mathrm{e}}} .
\end{aligned}
$$

From the definition $\boldsymbol{e}(t)=x(t)-\hat{x}(t),(24)$ can be expressed as:

$$
\lim _{t \rightarrow \infty}\|x(t)\|=\frac{M_{1}\|\boldsymbol{D}\| L}{\lambda_{\mathrm{e}}}\left(1+\frac{M_{2} \| \hat{S} \text { OOC } \|}{\lambda_{\mathrm{o}}}\right) .
$$

Therefore, when LMI (20) is satisfied, the SMC (13) can guarantee the asymptotic stability of the closed-loop system (18). Moreover, the system state is within the region of the equilibrium with the radius $\frac{M_{1}\|\boldsymbol{D}\| L}{\lambda_{\mathrm{e}}}\left(1+\frac{M_{2} \| \hat{\text { SOC } \|}}{\lambda_{\mathrm{o}}}\right)$.

\subsection{Optimize the Control Parameters}

Considering the SMC (13), when selecting larger values of $\mu$ and $\beta$, the system state can reach the sliding mode surface faster. At the same time, it is able to reduce the time of load frequency oscillation. However, when the system state reaches the sliding surface with a high speed, the deviation of the load frequency will become larger simultaneously.

In addition, the Lyapunov function of the system (19) is designed as:

$$
\boldsymbol{V}(t)=\boldsymbol{e}^{\mathrm{T}}(t) \boldsymbol{P}_{1} \boldsymbol{e}(t),
$$

Taking the derivative of (26), one has

$$
\dot{\boldsymbol{V}}(t)=\boldsymbol{e}^{\mathrm{T}}(t)\left(\operatorname{sym}\left(\boldsymbol{P}_{1}(\boldsymbol{A}-\boldsymbol{O C})\right)+\boldsymbol{Q}_{1}\right) \boldsymbol{e}(t) .
$$

According to the Lyapunov stability theory, when the eigenvalue of $Q_{1}$ take larger values, it will enable the error system to converge quickly and improve the dynamic performance of the error system. However, a larger value of the eigenvalue $\lambda_{e}$ of $Q_{1}$ will contribute to larger parameter for the observer gain $O$. It is seen from the observer

$$
\dot{\hat{x}}(t)=(A-B K) \hat{x}(t)+\hat{S} O C e(t)
$$


that the dynamic performance of the observer will be damaged. To improve the dynamic performance of the closed-loop system, a compromise solution is proposed.

Combining (17) and (26), it can be obtained that

$$
\begin{aligned}
\dot{\boldsymbol{V}}(t) & \leq-\boldsymbol{e}^{\mathrm{T}}(t) \boldsymbol{Q}_{1} \boldsymbol{e}(t) \\
& \leq-\lambda_{\min }\left(\boldsymbol{Q}_{1}\right)\|\boldsymbol{e}(t)\|^{2} .
\end{aligned}
$$

Considering the LMIs (20), with the increasing eigenvalues of $Q_{1}, \lambda_{e}$ also increases. The parameters $Q_{2}$ and $\lambda_{o}$ have the same property. Therefore, by optimizing the trace of $Q_{1}$ and $Q_{2}$, the larger $\lambda_{e}$ and $\lambda_{o}$ can be obtained. At the same time, the smaller value of $\|\hat{S O C}\|$ can be guaranteed by optimizing the trace of the matrix $O C$.

From the discussion above, the matrix $O, K$ and $P_{1}, P_{2}, Q_{1}, Q_{2}$ can be solved by the following optimization problem:

$$
\begin{array}{ll}
\max _{\boldsymbol{Q}_{1}, \boldsymbol{Q}_{2}} & \operatorname{tr}\left(\boldsymbol{Q}_{1}\right), \operatorname{tr}\left(\boldsymbol{Q}_{2}\right) ; \min _{O} \operatorname{tr}(\boldsymbol{O C}) \\
\text { s.t. } & \operatorname{sym}\left(\boldsymbol{P}_{1}\left(\boldsymbol{A}^{(k)}-\boldsymbol{O C}\right)\right)+\boldsymbol{Q}_{1}<0, \\
& \operatorname{sym}\left(\left(\boldsymbol{A}^{(k)}-\boldsymbol{B}^{(k)} \boldsymbol{K}\right) \boldsymbol{P}_{2}\right)+\boldsymbol{Q}_{2}<0,
\end{array}
$$

where $k=1,2, \ldots, M$.

Through the above analysis, in the process of optimization of the controller parameters, we have supplemented the maximum values of the trace of $Q_{1} Q_{2}$ and the maximum value of the trace of $O C$, denoted by $\max \operatorname{tr}\left(Q_{1}\right), \operatorname{tr}\left(Q_{2}\right)$ and $\min \operatorname{tr}(\boldsymbol{O C})$, respectively. In this case, we can use the minimum value of $-\operatorname{tr}\left(Q_{1}\right),-\operatorname{tr}\left(Q_{2}\right)$ equivalent to the maximum value of $\operatorname{tr}\left(Q_{1}\right), \operatorname{tr}\left(Q_{2}\right)$. In this way, after combining $\min \operatorname{tr}(\boldsymbol{O C})$, we use the "mincx" function in the LMI toolbox to solve the controller parameters.

\section{Simulation Analysis}

In this section, we consider the interconnected power systems with two regions containing wind power and EVs. The designed SMC is applied to the system model for LFC. For the conventional PID (Proportion Integral Differential) controller, by comparing the systems contain EVs or not, the simulations are given.

Assume the system subjected to perturbations from changes in load demand at $t \geq 0$. The magnitudes are $\Delta P_{\mathrm{d} 1}=0.01 \mathrm{pu}$ and $\Delta P_{\mathrm{d} 2}=0.02 \mathrm{pu}$, respectively. In the two regions, the turbine scale factor is $\alpha_{\mathrm{g} 1}=0.9, \alpha_{\mathrm{g} 2}=0.6$, EVs scale factor is $\alpha_{\mathrm{e} 1}=0.1, \alpha_{\mathrm{e} 2}=0.4$, and wind power is $\Delta P_{\mathrm{w}-1}=0.04 \mathrm{pu}, \Delta P_{\mathrm{w}-2}=0.05 \mathrm{pu}$. According to the topology and the analysis in the mathematical model, the generator units are equivalent in each region in the simulation process. After idealized values for the model parameters, the system parameters of the two areas are shown in Table 1.

Table 1. Regional system parameters.

\begin{tabular}{ccc}
\hline Parameters & Region $\mathbf{1}$ & Region 2 \\
\hline$T_{\mathrm{ch} i}$ & $0.35 \mathrm{~s}$ & $0.352 \mathrm{~s}$ \\
$T_{\mathrm{g} i}$ & $0.08 \mathrm{~s}$ & $0.08 \mathrm{~s}$ \\
$R_{i}$ & $5 \mathrm{~Hz} / \mathrm{up}$ & $5 \mathrm{~Hz} / \mathrm{put}$ \\
$\beta_{i}$ & $0.4 \mathrm{~Hz} / \mathrm{pu}$ & $0.4 \mathrm{~Hz} / \mathrm{pu}$ \\
$D_{i}$ & $0.02 \mathrm{~Hz} / \mathrm{pu}$ & $0.01 \mathrm{~Hz} / \mathrm{pu}$ \\
$M_{i}$ & $0.3 \mathrm{pu} \cdot \mathrm{s}$ & $0.3 \mathrm{pu} \cdot \mathrm{s}$ \\
$T_{\mathrm{e} i}$ & $1 \mathrm{~s}$ & $1 \mathrm{~s}$ \\
$T_{\mathrm{WTG} i}$ & $1.5 \mathrm{~s}$ & $1.52 \mathrm{~s}$ \\
$T_{i j}$ & $0.2 \mathrm{pu} / \mathrm{rad}$ & $0.22 \mathrm{pu} / \mathrm{rad}$ \\
\hline
\end{tabular}




\subsection{PID Control}

The PID controller parameters are shown in Table 2. The simulation results of the two regional power systems are shown in Figure 3.

Table 2. PID controller parameters.

\begin{tabular}{ccc}
\hline Symbol & Region $\mathbf{1}$ & Region 2 \\
\hline$K_{\mathrm{p}}$ & -0.6 & -0.6 \\
$K_{\mathrm{i}}$ & -1 & -1.1 \\
$K_{\mathrm{d}}$ & -1.2 & -1.3 \\
\hline
\end{tabular}

As shown in Figure 4, the PID controller is able to stabilize the load frequency deviation at $t=50 \mathrm{~s}$. It is shown from the simulation that the system containing EVs can take less time to achieve stability. In addition, the system containing EVs also has less fluctuations. The effectiveness of utilizing energy storage of EVs to participate in frequency control is demonstrated.

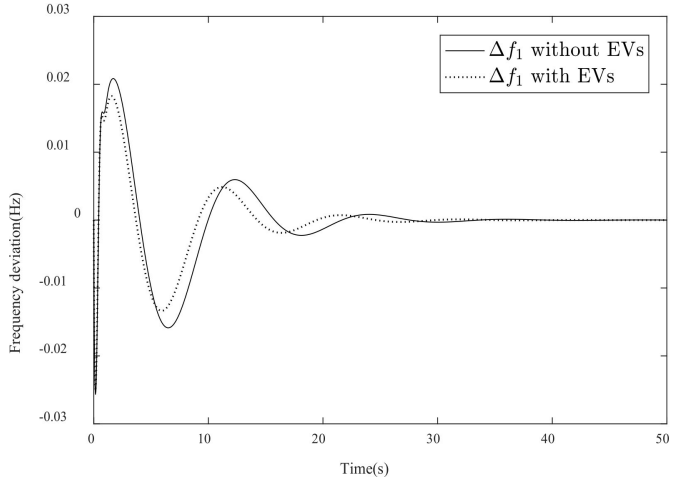

(a)

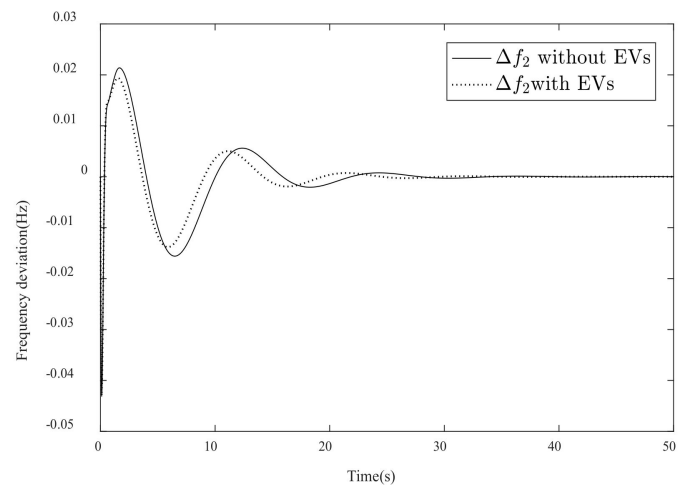

(b)

Figure 4. (a) Frequency deviation $\Delta f_{i}$ with and without EVs in region 1 ; (b) frequency deviation $\Delta f_{i}$ with and without EVs in region 2.

\subsection{Integral Sliding Mode Control}

Next, the SMC will be used for simulation. The matrices in the two area controllers are as follows:

$S_{1}=\left[\begin{array}{lllllll}0 & 0 & 1 & 0 & 0 & 0 & 17\end{array}\right] S_{2}=\left[\begin{array}{lllllll}0 & 0 & 1 & 0 & 0 & 0 & 18\end{array}\right], \mu_{1}=5, \mu_{2}=9, v_{1}=0.1, v_{2}=0.1$.

By solving (20), the sliding surface for region 1 and region 2 are obtained as:

$$
\begin{aligned}
& s_{1}(t)=53.6373 \Delta \hat{f}_{1}-85.3485 \Delta \hat{P}_{\mathrm{m} 1}-37.1822 \Delta \hat{P}_{\mathrm{v} 1}+\Delta \dot{\hat{P}}_{\mathrm{v} 1}+-23.6768 \Delta \hat{P}_{\mathrm{tie}-1}-40.4814 \int \hat{\delta}_{1}+99.7098 \Delta \hat{P}_{\mathrm{WTG} 1}-114.8640 \Delta \hat{P}_{\mathrm{e} 1}+17 \Delta \dot{\hat{P}}_{\mathrm{e} 1} \\
& s_{2}(t)=56.7137 \Delta \hat{f}_{1}-89.5246 \Delta \hat{P}_{\mathrm{m} 1}-39.0279 \Delta \hat{P}_{\mathrm{v} 1}+\Delta \dot{\hat{P}}_{\mathrm{v} 1}+-24.2960 \Delta \hat{P}_{\mathrm{tie}-1}-42.6785 \int \hat{\delta}_{1}+104.6242 \Delta \hat{P}_{\mathrm{WTG} 1}-120.5132 \Delta \hat{P}_{\mathrm{e} 1}+18 \Delta \dot{\hat{P}}_{\mathrm{e} 1},
\end{aligned}
$$

The sliding controller for region 1 and region 2 are obtained as:

$u_{1}(t)=-3.9488 \Delta \hat{f}_{1}-6.5906 \Delta \hat{P}_{\mathrm{m} 1}-1.9060 \Delta \hat{P}_{\mathrm{v} 1}+1.8283 \Delta \hat{P}_{\text {tie }-1}-3.1260 \int \hat{\delta}_{1}-7.6996 \Delta \hat{P}_{\mathrm{WTG} 1}-7.5571 \Delta \hat{P}_{\mathrm{e} 1}-1.2406\left(\Delta f_{1}-\Delta \hat{f}_{1}\right)$

$+0.0186\left(\int \delta_{1}-\int \hat{\delta}_{1}\right)-0.2066 s(t)-0.0041 \operatorname{sgn}(s(t))$,

$u_{2}(t)=-4.1543 \Delta \hat{f}_{2}-6.8601 \Delta \hat{P}_{\mathrm{m} 2}-2.0328 \Delta \hat{P}_{\mathrm{v} 2}+1.8618 \Delta \hat{P}_{\text {tie }-2}-3.2704 \int \hat{\delta}_{2}-8.0172 \Delta \hat{P}_{\mathrm{WTG} 2}-7.8554 \Delta \hat{P}_{\mathrm{e} 2}-1.3495\left(\Delta f_{2}-\Delta \hat{f}_{2}\right)$ $+0.0110\left(\int \delta_{2}-\int \hat{\delta}_{2}\right)-0.3704 s(t)-0.0041 \operatorname{sgn}(s(t))$,

where $\Delta \hat{f}_{i}$ is the estimation of $\Delta f_{i}, \Delta \hat{P}_{\mathrm{mi}}$ is the estimation of $\Delta P_{\mathrm{mi}}, \Delta \hat{\mathrm{P}}_{\mathrm{vi}}$ is the estimation of $\Delta P_{\mathrm{vi}}, \Delta \hat{P}_{\text {tie }-i}$ is the estimation of $\Delta P_{\text {tie }-i}, \int \hat{\delta}_{i}$ is the estimation of $\int \delta_{i}, \Delta \hat{P}_{\mathrm{e} i}$ is the estimation of $\Delta P_{\mathrm{e} i}, \Delta \hat{P}_{\mathrm{WTG} i}$ is the estimation of $\Delta P_{\mathrm{WTG} i}$. 
The simulation results under the SMC are shown in Figures 5-7.

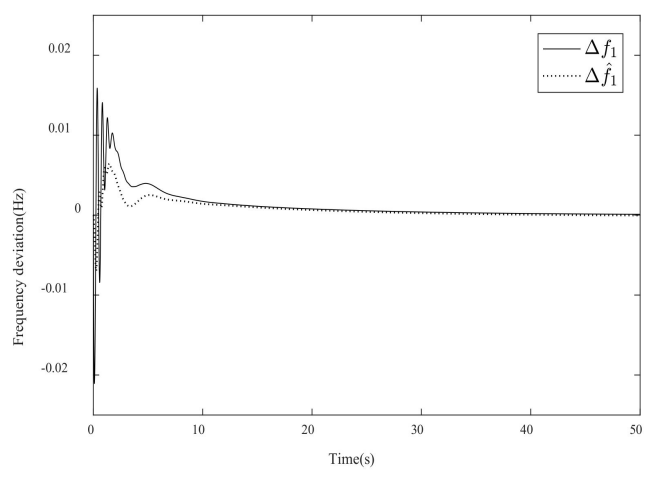

(a)

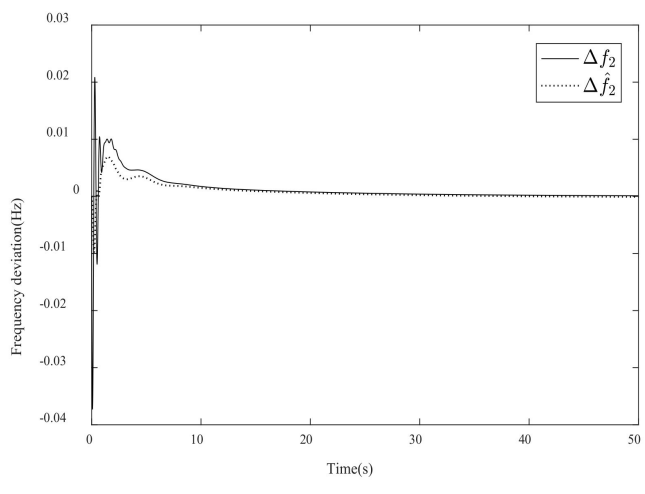

(b)

Figure 5. (a) Frequency deviation $\Delta f_{i}$ and observed frequency deviation $\Delta \hat{f}_{i}$ in region 1 ; (b) frequency deviation $\Delta f_{i}$ and observed frequency deviation $\Delta \hat{f}_{i}$ in region 2 .

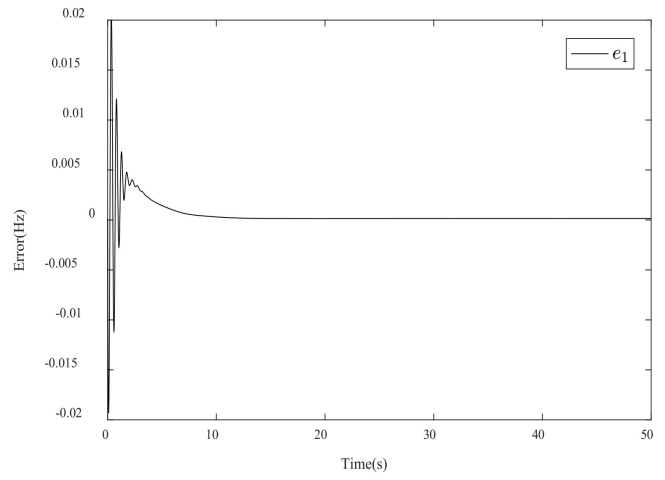

(a)

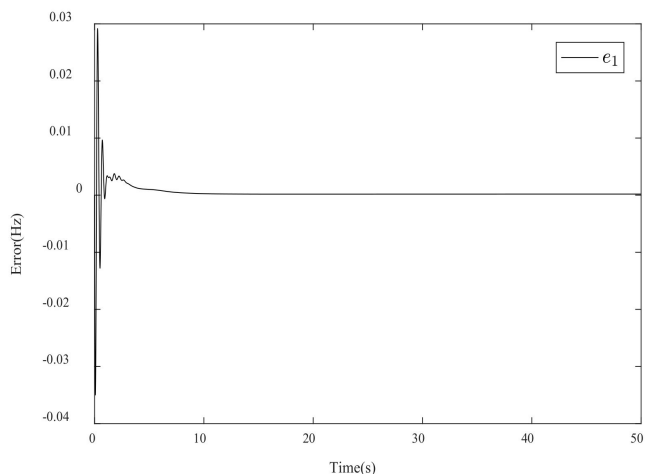

(b)

Figure 6. (a) Estimated error of frequency deviation $e_{1}$ in region $1 ;(\mathbf{b})$ estimated error of frequency deviation $e_{1}$ in region 2.

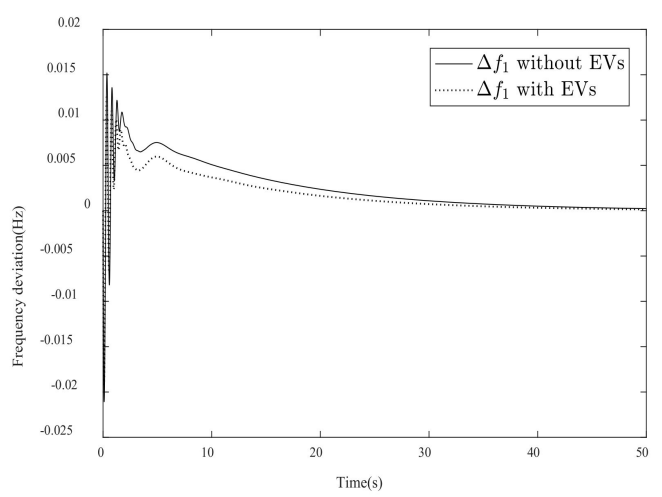

(a)

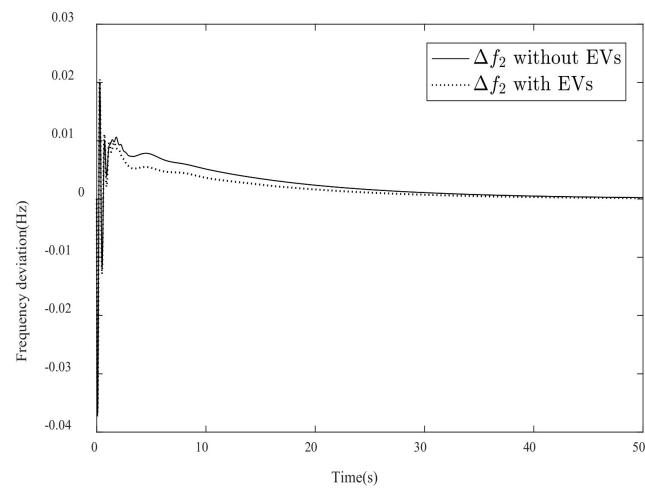

(b)

Figure 7. (a) Frequency deviation $\Delta f_{i}$ with and without EVs in region 1 ; (b) frequency deviation $\Delta f_{i}$ with and without EVs in region 2.

As can be seen from Figure 5, the integrated SMC enables the stabilization of the load frequency deviation in both regions. Additionally, from Figure 5, it is shown that the observed value $\Delta \hat{f}_{i}$ tracks the parameter $\Delta f_{i}$ effectively. Further combined with Figure 6, the observer proposed can achieve the estimation of the unknown state system. 
In SMC, we consider the LFC problem of system including EV and without EV. To compare the system performance between PID and SMC, we summarized the data from the simulation results, which is shown in Table 3, compared with the results of PID, SMC designed can reach stability in shorter time. Also, the fluctuation phenomenon is smoother. In addition, through Figure 7, it is seen that SMC reduce the stable time for system containing EVs. According to the above analysis, better control performance of the $\mathrm{SMC}$ and the effectiveness of EVs participation in frequency control is verified.

Table 3. Summary of simulation results data of sliding mode controller (SMC) and PID.

\begin{tabular}{llcccc}
\hline & & \multicolumn{2}{c}{ PID } & \multicolumn{2}{c}{ SMC } \\
& & $\begin{array}{c}\text { Overshoot } \\
\mathbf{( H z )}\end{array}$ & $\begin{array}{c}\text { Stable } \\
\text { Time(s) }\end{array}$ & $\begin{array}{c}\text { Overshoot } \\
\mathbf{( H z )}\end{array}$ & $\begin{array}{c}\text { Stable Time } \\
\mathbf{( s )}\end{array}$ \\
\hline \multirow{2}{*}{ without EVs } & region 1 & 0.0215 & 49.70 & 0.0152 & 45.20 \\
\multirow{2}{*}{ with EVs } & region 2 & 0.0219 & 48.60 & 0.0202 & 43.50 \\
& region 1 & 0.0190 & 47.60 & 0.0130 & 40.30 \\
& region 2 & 0.0205 & 45.30 & 0.0212 & 39.80 \\
\hline
\end{tabular}

\subsection{Optimize the $S M C$}

In this part, the simulation of the optimized SMC will be carried out. The matrix in the controllers is selected as follows:

$$
S_{1}=\left[\begin{array}{lllllll}
0 & 0 & 1 & 0 & 0 & 0 & 17
\end{array}\right], S_{2}=\left[\begin{array}{lllllll}
0 & 0 & 1 & 0 & 0 & 0 & 18
\end{array}\right], \mu_{1}=5, \mu_{2}=9, v_{1}=0.1, v_{2}=0.1
$$

By solving (29), the optimized sliding surfaces of region 1 and region 2 are obtained as follows:

$$
\begin{aligned}
& s_{1}(t)=2.4957 \Delta \hat{f}_{1}-0.0002 \Delta \hat{P}_{\mathrm{m} 1}-12.5012 \Delta \hat{P}_{\mathrm{v} 1}+\Delta \dot{\hat{P}}_{\mathrm{v} 1}-0.0512 \Delta \hat{P}_{\text {tie }-1}-0.9015 \int \hat{\delta}_{1}+0.0005 \Delta \hat{P}_{\mathrm{WTG} 1}+17.0251 \Delta \hat{P}_{\mathrm{e} 1}+17 \Delta \dot{\hat{P}}_{\mathrm{e} 1}, \\
& s_{2}(t)=2.5102 \Delta \hat{f}_{1}-0.0004 \Delta \hat{P}_{\mathrm{m} 1}+12.4598 \Delta \hat{\mathrm{P}}_{\mathrm{v} 1}-\Delta \dot{\hat{P}}_{\mathrm{v} 1}-0.0450 \Delta \hat{P}_{\text {tie }-1}-0.8845 \int \hat{\delta}_{1}-0.0004 \Delta \hat{P}_{\mathrm{WTG} 1}+17.9895 \Delta \hat{P}_{\mathrm{e} 1}+18 \Delta \dot{\hat{P}}_{\mathrm{e} 1} .
\end{aligned}
$$

The optimized sliding controllers of region 1 and region 2 are obtained as follows:

$u_{1}(t)=-0.2445 \Delta \hat{f}_{1}-0.0152 \Delta \hat{P}_{\mathrm{m} 1}-0.0814 \Delta \hat{P}_{\mathrm{v} 1}+0.0054 \Delta \hat{P}_{\mathrm{tie}-1}-0.0907 \int \hat{\delta}_{1}-1.3501 \Delta \hat{P}_{\mathrm{WTG} 1}-0.1491 \Delta \hat{P}_{\mathrm{e} 1}-4.5103\left(\Delta f_{1}-\Delta \hat{f}_{1}\right)$ $-0.0021\left(\int \delta_{1}-\int \hat{\delta}_{1}\right)-0.2066 s(t)-0.0041 \operatorname{sgn}(s(t))$,

$u_{2}(t)=-1.2105 \Delta \hat{f}_{2}-0.0135 \Delta \hat{P}_{\mathrm{m} 2}-0.1437 \Delta \hat{P}_{\mathrm{v} 2}+0.0098 \Delta \hat{P}_{\mathrm{tie}-2}-0.1592 \int \hat{\delta}_{2}-0.7510 \Delta \hat{P}_{\mathrm{WTG} 2}-0.2619 \Delta \hat{P}_{\mathrm{e} 2}-2.5012\left(\Delta f_{2}-\Delta \hat{f}_{2}\right)$ $-0.1511\left(\int \delta_{2}-\int \hat{\delta}_{2}\right)-0.3704 s(t)-0.0041 \operatorname{sgn}(s(t))$,

The simulation results under the optimized SMC are shown in Figures 8-10.

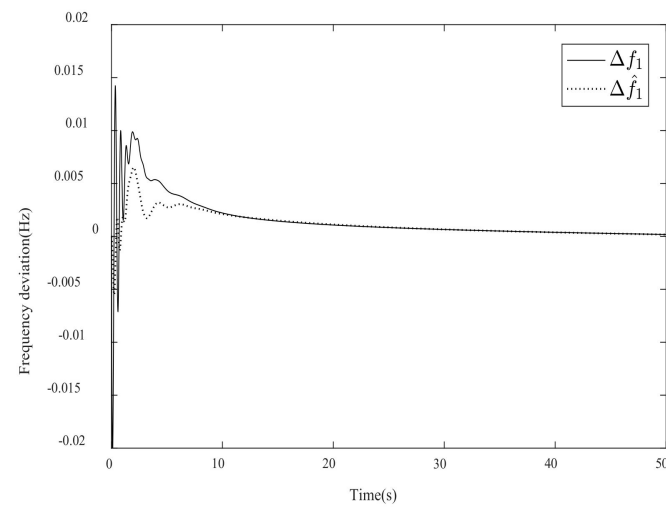

(a)

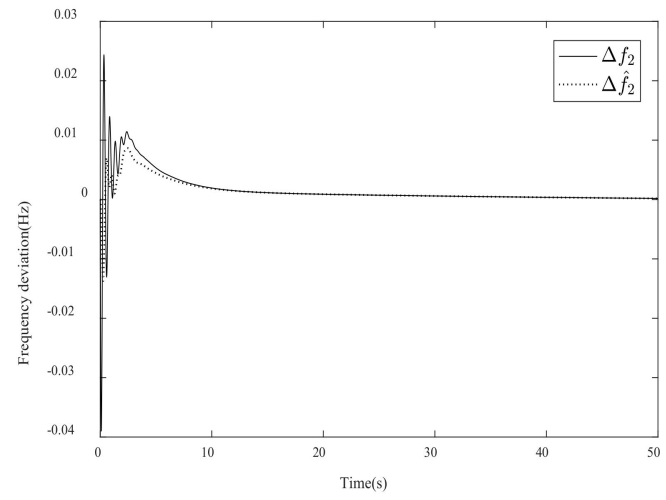

(b)

Figure 8. (a) Frequency deviation $\Delta f_{i}$ and observed frequency deviation $\Delta \hat{f}_{i}$ in region 1 ; (b) frequency deviation $\Delta f_{i}$ and observed frequency deviation $\Delta \hat{f}_{i}$ in region 2 . 


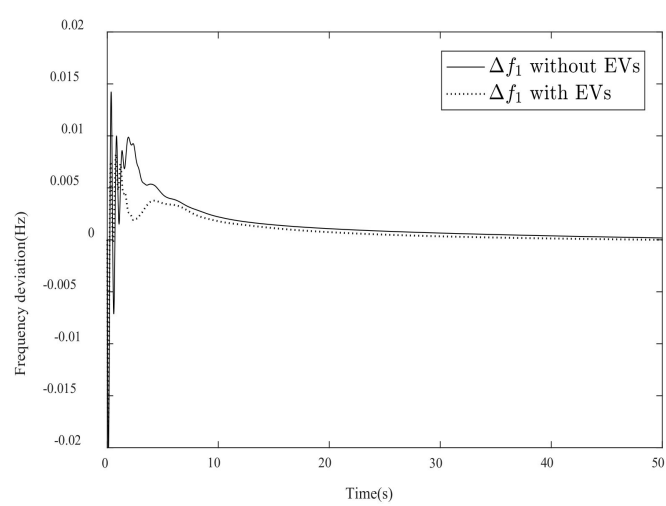

(a)

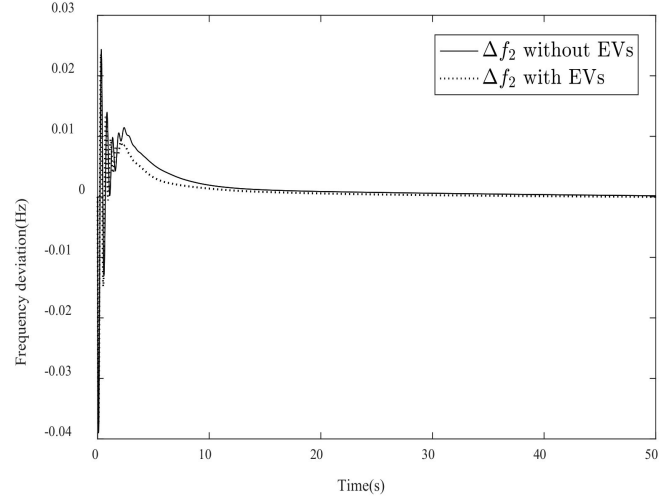

(b)

Figure 9. (a) Frequency deviation $\Delta f_{i}$ with and without EVs in region 1 ; (b) frequency deviation $\Delta f_{i}$ with and without EVs in region 2.

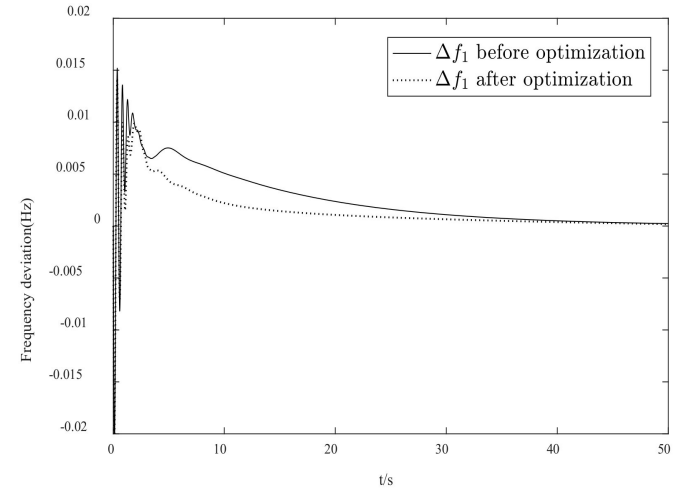

(a)

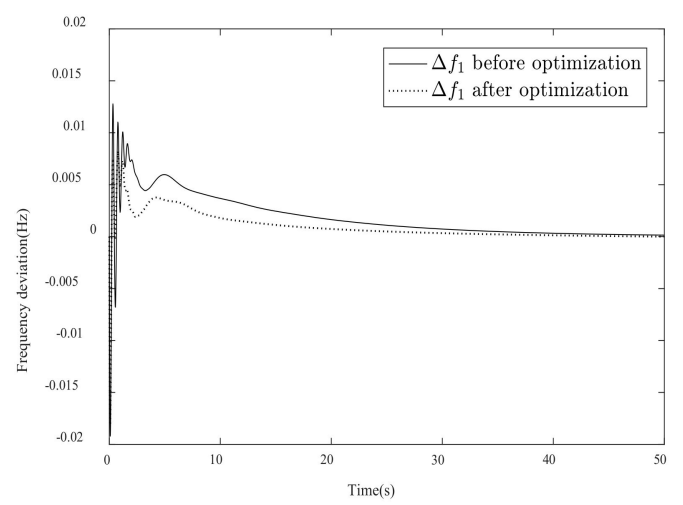

(c)

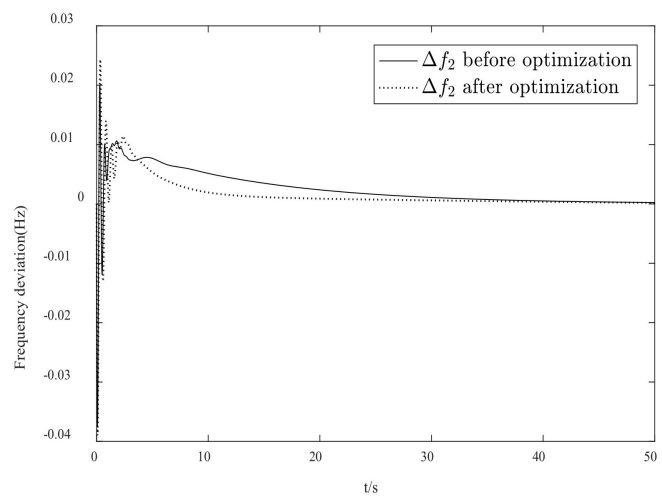

(b)

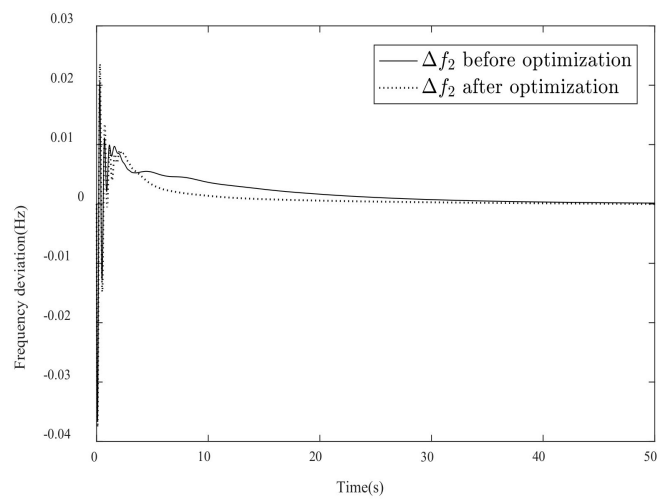

(d)

Figure 10. (a) Comparison of frequency deviation $\Delta f_{i}$ without EVs in region 1. (b) Comparison of frequency deviation $\Delta f_{i}$ without EVs in region 2. (c) Comparison of frequency deviation $\Delta f_{i}$ with EVs in region 1. (d) Comparison of frequency deviation $\Delta f_{i}$ with EVs in region 2 .

In this section, we first verify that the optimized observer can observe the state of the system, which is shown in Figure 8. According to Figure 8, it is shown that the optimized SMC can enable the load frequency deviation to reach stable values. Similarly, after optimization, the control performance was verified in system with and without EVs. By comparing the simulation results in Figure 9, for system containing EVs, it can achieve stability with smaller fluctuations and shorter time. 
To further verify the effectiveness of the optimized SMC, we compare the control performance of the controller before and after the optimization. Simulations are performed in system with and without EVs, the results are shown in Figure 10. To compare the results, we have summarized the data from the simulation results, as shown in Table 4.

Table 4. Summary of simulation results data of SMC.

\begin{tabular}{|c|c|c|c|c|c|}
\hline & & \multicolumn{2}{|c|}{ Before Optimization } & \multicolumn{2}{|c|}{ After Optimization } \\
\hline & & $\begin{array}{l}\text { Overshoot } \\
(\mathrm{Hz})\end{array}$ & $\begin{array}{c}\text { Stable } \\
\text { Time(s) }\end{array}$ & $\begin{array}{l}\text { Overshoot } \\
(\mathrm{Hz})\end{array}$ & $\begin{array}{c}\text { Stable Time } \\
\text { (s) }\end{array}$ \\
\hline \multirow{2}{*}{ without EVs } & region 1 & 0.0152 & 45.20 & 0.0140 & 35.40 \\
\hline & region 2 & 0.0202 & 43.50 & 0.0242 & 37.30 \\
\hline \multirow{2}{*}{ with EVs } & region 1 & 0.0130 & 40.30 & 0.0080 & 31.45 \\
\hline & region 2 & 0.0212 & 39.80 & 0.0235 & 30.70 \\
\hline
\end{tabular}

Through comparing the simulation results of in Figure 10a,b, in the case of the system without EVs, the parameter optimized SMC can make $\Delta f_{i}$ stable in a shorter time. Similarly, in the case of the system containing EVs, the same conclusion can be obtained from the simulation results of in Figure 10c,d. From the above analysis, the effectiveness and the superiority of the parameter optimized controller are also further verified.

\section{Conclusions}

This paper aims to reduce the frequency fluctuation of systems with wind power and EVs. Through the above simulation analysis, we have solved the impact of monitoring equipment limitations, disturbances, and wind power uncertainty on the system. Further, the controller parameters have been optimized by combining the Lyapunov stability principle. The subsequent conclusions are drawn from the presented work:

1. The observer designed in this paper can realize the observation of the system state. Moreover, the observer-based SMC is designed in this paper. It not only realizes the stability in a certain time, but also has better control performance.

2. Through the simulation results, the EVs has better suppression effect on the load frequency fluctuation of the power system. Thus, the effectiveness of the view that EVs as storage participate in frequency control is also verified.

3. By comparing the figures before and after optimization, it is shown that the optimized SMC can enable the system load frequency reach stable value in shorter time, which improves the robustness of the interconnected power system.

With the above analysis, this paper realizes the LFC for the system with wind power and EVs. Also, we found that the energy storage of EVs can effectively participate in the frequency control. It is noted that through the simulation results of the system with two regions interconnected, we have verified the research objectives of this paper. In the future research, simulations of multiple region interconnections may be further considered.

Author Contributions: All authors contributed to the research in this paper. Y.L. conceived the methodology and model of the research system of this article. Z.W. performed simulation verification and analyzed the data. Z.Y. finished the original draft of the article. W.Y. edited and reviewed the article. All authors have read and agreed to the published version of the manuscript.

Funding: This work is supported by the National Natural Science Foundation of China (grant no. 61803253, 61973209).

Institutional Review Board Statement: Not applicable.

Informed Consent Statement: Not applicable.

Conflicts of Interest: The authors declare no conflict of interest. 


\section{Appendix A}

Table A1. Nomenclature of system model.

\begin{tabular}{cccc}
\hline$\Delta f_{i}$ & frequency deviation & $D_{i}$ & load damping coefficient \\
\hline$\Delta P_{\mathrm{m} i}$ & generator power deviation & $M_{i}$ & inertia constant \\
$\Delta P_{\mathrm{v} i}$ & control valve position deviation & $T_{\mathrm{g} i}$ & governor time constant \\
$\Delta P_{\mathrm{tie}-i}$ & tie-line power exchange & $T_{\mathrm{ch} i}$ & turbine time constant \\
$\delta_{i}$ & area control deviation & $R_{i}$ & governor droop characteristic \\
$\Delta P_{\mathrm{WTG} i}$ & wind turbine power deviation & $\beta_{i}$ & frequency deviation factor \\
$\Delta P_{\mathrm{W}-i}$ & power of wind & $\Delta P_{\mathrm{d} i}$ & load demand disturbance \\
$\Delta P_{\mathrm{e} i}$ & EVs output power deviation & $\alpha_{\mathrm{g} i}$ & turbine proportionality factor \\
$k_{\mathrm{e} i}$ & EVs gain factor & $\alpha_{\mathrm{e} i}$ & EVs proportionality factor \\
$T_{\mathrm{e} i}$ & EVs time instants & & \\
\hline
\end{tabular}

\section{References}

1. Ourahou, M.; Ayrir, W.; Hassouni, B.E. Review on smart grid control and reliability in presence of renewable energies: Challenges and prospects. Math. Comput. Simul. 2020, 167, 19-31. (In Chinese) [CrossRef]

2. Zhou, X.X.; Lu, Z.X.; Liu, Y.M. Development models and key technologies of future grid in China. Proc. CSEE 2014, 23, 4999-5008. (In Chinese)

3. Li, Y.P.; Yan, Z.; Jia, Y.B. Review of multi-area interconnected power system reliability evaluation. East China Electr. Power 2009, 37, 1848-1854. (In Chinese)

4. Yao, J.G.; Yang, S.C.; Shan, M.H. Reflections on operation supporting system architecture for future interconnected power grid. Autom. Electr. Power Syst. 2012, 37, 52-59. (In Chinese)

5. Bai, H.B.; Wang, R.H. Influence of the grid-connected wind farm on power quality. Proc. CSU-EPSA 2012, 24, 120-124. (In Chinese)

6. Li, J.H.; Zuo, J.J.; Wang, S. Analysis and assessment of operation risk for power system with large-scale wind power integration. Power Syst. Technol. 2016, 42, 3503-3513. (In Chinese)

7. Tang, X.J.; Cai, J.C.; Ma, S.Y. Effects of DFIG wind power grid-connection on frequency response of power system. Proc. CSU-EPSA 2020, 32, 37-43, 62. (In Chinese)

8. Yang, D.Y.; Cai, G.W. Decentralized model predictive control-based load frequency control for high wind power penetrated power system. Proc. CSEE 2015, 35, 583-591. (In Chinese)

9. Han, Y.H.; Ma, C.; Zhu, Y.Z. Load frequency control for power systems with wind energy. Control Eng. China 2018, 25, $2046-2051$. (In Chinese)

10. Yang, C.; Yao, W.; Wen, J.Y. Event triggering based robust control of load frequency for interconnected power grid with wind power. Autom. Electr. Power Syst. 2018, 42, 57-64, 207-211. (In Chinese)

11. Yang, L.; Sun, Y.Z.; Xu, J. Adaptive load frequency control of wind power system based on online reinforcement learning. Autom. Electr. Power Syst. 2020, 44, 74-89. (In Chinese)

12. Scarabaggio, P.; Grammatico, S.; Carli, R. Distributed demand side management with stochastic wind power forecasting. IEEE Trans. Control Syst. Technol. 2021, 1-16. [CrossRef]

13. Huang, Y.; Liu, J.Y.; Chen, J.R. Load frequency control considering vehicle to grid. Autom. Electr. Power Syst. 2012, 36, 24-28. (In Chinese)

14. Zhang, Q.; Zhou, L.; Zhou, L.W. Load frequency control considering charging and discharging static frequency characteristics of electric vehicles. Autom. Electr. Power Syst. 2014, 38, 74-80. (In Chinese)

15. Pan, Y.T.; Tan, W. Load frequency control considering vehicle to grid based on linear active disturbance rejection control. Control Eng. China 2018, 25, 1226-1231. (In Chinese)

16. Yang, J.W.; Dong, H.Z.; Liao, K. Joint optimization of load frequency control considering auxiliary frequency regulation of electric vehicles. Power Syst. Technol. 2019, 39, 200-206. (In Chinese)

17. Shang-Guan, X.C.; He, Y.; Zhang, C. Sampled-data based discrete and fast load frequency control for power systems with wind power. Appl. Energy 2020, 259, 114202. [CrossRef]

18. Sperstad, I.B.; Korpås, M. Energy storage scheduling in distribution systems considering wind and photovoltaic generation uncertainties. Energies 2019, 12, 1231. [CrossRef]

19. Aravindh, D.; Sakthivel, R.; Kaviarasan, B. Design of observer-based non-fragile load frequency control for power systems with electric vehicles. ISA Trans. 2019, 91, 21-31. [CrossRef] [PubMed] 